\title{
Paulownia tomentosa flower polysaccharide as an effective immunopotentiator to enhance immune responses for Newcastle disease vaccine in mice
}

\author{
Xiaolan Chen ${ }^{1 *}$, Junjie Jin ${ }^{2}$, Fuxing Hao ${ }^{1}$, Haifeng Yang ${ }^{1}$, Hongxiang Sun $^{3}$, Chunmao Jiang ${ }^{1}$ \\ ${ }^{1}$ Jiangsu Agri-animal Husbandry Vocational College, Taizhou, China; ${ }^{2}$ Wenzhou Vocational College of Science and \\ Technology, Wenzhou, China; ${ }^{3}$ Zhejiang University, Hangzhou, China
}

*Corresponding author: Xiaolan Chen, Jiangsu Agri-animal Husbandry Vocational College, Taizhou, Jiangsu Province, China. Email: cxl7972563@163.com

Received: 23 July 2021; Accepted: 27 September 2021; Published: 2 November 2021

(C) 2021 Codon Publications

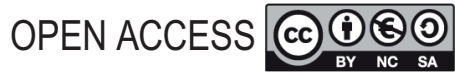

PAPER

\begin{abstract}
To investigate the immunomodulatory activity and explore the mechanism of Paulownia tomentosa flower polysaccharides (PTFP). PTFP was orally administrated to mice for seven successive days before and after Newcastle disease vaccination. The results demonstrated that compared with the vaccine control (VC) group, PTFP enhanced the inhibition of hemagglutination assay antibody titers, promoted the antigen-specific immunoglobulin (Ig)G, IgG1, IgG2a, and IgG2b antibodies responses, enhanced proliferation of spleen T and B lymphocytes, increased the secretions of interferon- $\gamma$ and interleukin-10 cytokines of spleen lymphocytes, and promoted the activation of natural killer cells. Therefore, PTFP, as an effective immunopotentiator, could induce a mixed T-helper (Th)1 and Th2 immune responses and an innate immune response.
\end{abstract}

Keywords: immune responses, immunopotentiator, Newcastle disease vaccine, Paulownia tomentosa flower polysaccharides

\section{Introduction}

In recent years, polysaccharides from Chinese medicinal herbs have drawn more attention because of their effective immune enhancement, favorable safety, and excellent biocompatibility (Sun et al., 2018; Tang et al., 2019; Chen et al., 2020). Various polysaccharides, such as Astragalus polysaccharide, Lentinan, Angelica sinensis polysaccharide, and Lycium barbarum polysaccharide, have been proved to possess potent immunomodulatory activity (Su et al., 2014; Wang et al., 2016; Sun et al., 2018; Chen et al., 2020; Ren et al., 2021). The Paulownia tomentosa ( $P$. tomentosa), as Chinese herbal medicine, has been widely used to treat stomach disorders, diarrhea, gonorrhea, erysipelas, hypertension, enteritis, tonsillitis, bronchitis, and dysentery (Dai et al., 2015; Liu et al., 2017; Lee et al., 2018; Wang et al., 2019). Recent researches have proved that $P$. tomentosa possesses various pharmacological activities, such as antibacterial, anti-inflammatory, antiphlogistic, antitussive, antiasthmatic, immunomodulatory, antioxidant, antiviral, and anticholinesterase activities (Dai et al., 2015; Liu et al., 2017; Wang et al., 2019). P. tomentosa flower polysaccharides (PTFP), which are extracted from the P. tomentosa flower, are the main water-soluble component of this flower. It has been reported that PTFP could serve as a new immunopotentiator to enhance humoral and cellular immune responses (Wang et al., 2019).

Newcastle disease (ND), one of the most contagious and devastating diseases in the poultry industry around the world, is caused by an avian paramyxovirus type 1 serotype of the genus Avulavirus in the Paramyxoviridae family (Zhai et al., 2011(a); Yuan et al., 2020; Chen et al., 2021). This disease reduced the production of eggs, led to respiratory and central nervous infections, death 
of poultry, and caused an immense economic loss in the poultry industry ( Ma et al., 2019; Yuan et al., 2020). Vaccination is the most effective and productive approach to prevent and control the spread of ND (Yang et al., 2020). The commercial vaccines available were live or inactivated virus-based vaccines. So to enhance immune responses for the ND vaccine, immunopotentiators or adjuvants were commonly required (Zhai et al., 2011(b); Ma et al., 2019; Yuan et al., 2020 ).

The immunomodulatory activity of PTFP was determined in our previous study by orally administrating it into the ND-vaccinated chickens. . The in vivo experiment results demonstrated that PTFP could improve lymphocyte proliferation, increase antibody response, and enhance the secretion of interferon (IFN) $-\gamma$, indicating that PTFP has the potential to improve immune responses in ND-vaccinated chickens(Yang et al., 2019). However, the mechanism of PTFP enhancing immune responses for the ND vaccine is still unknown. Hence, to further investigate the immunomodulatory activity and mechanism of PTFP for improving the ND vaccine response. Mice used as the model animal were orally administrated with the immunopotentiator PTFP. Later they were immunized with ND-vaccine twice at an interval of 14 days. After the second dose of vaccination, inhibition of hemagglutination assay (IHA) titers against ND and antigen-specific immunoglobulin (Ig)G and isotypes (IgG1, IgG2a, and IgG2b) antibodies were determined. Meanwhile, for further immune responses evaluation, spleen lymphocytes proliferation, together with cytokines, and natural killer (NK) cells activity were measured.

\section{Materials and Methods}

\section{Materials}

Dried-cultured P. tomentosa flower were obtained from Bozhou Guoxin Pharmaceutical Co., Ltd (Anhui, China). Attenuated ND- vaccine (LaSota strain, No. 1170121) was purchased from Guangxi Liyuan Biotechnology Co., Ltd (China). Fetal bovine serum was obtained from Gibco (Carlsbad, CA). Roswell Park Memorial Institute (RPMI)1640 medium was purchased from HyClone, Logan, UT. Concanavalin A (Con A) and lipopolysaccharide (LPS) were obtained from Sigma-Aldrich (St. Louis, MO). The antigen and positive control sera used for the ND virus (NDV)-specific IHA was purchased from Qingdao YEBIO Biological Technology Co., Ltd (China). Horse radish peroxidase (HRP)-conjugated rabbit anti-mouse IgG antibody was purchased from Sigma-Aldrich. HRPconjugated goat anti-mouse IgG1, IgG2a, and IgG2b antibodies were obtained from Southern Biotechnology Associates (Birmingham, AL). IFN- $\gamma$ and interleukin
(IL)-10 enzyme-linked immunosorbent assay (ELISA) kits were obtained from BOSTER Biological Technology Co., Ltd (China). All other regents and chemicals were of analytical grade.

\section{Preparation of PTFP}

PTFP was prepared by water decoction and ethanol precipitation as previously described (Yang et al., 2019). In brief, dried cultured $P$. tomentosa flowers were extracted twice with boiling water for 2 hours and 1 hour, respectively. After filtration, the merged decoction was then condensed, and the suspension was precipitated with $95 \%$ ethanol four times for a total of 12 hours. The solution was then centrifuged and concentrated to a specific volume, dried under reduced pressure at $60^{\circ}$. The carbohydrate concentration (\%) of the total PTFP was 48 compared with D-glucose, and the luteolin and apigenin concentration (\%) of the total PTFP was 3.12 and 4.35, respectively.

\section{Cells and animals}

Human leukemia cell line K562 was obtained from Shanghai Institute of Cell Biology, Chinese Academy of Sciences.

Institute of Cancer Research mice (5-6 weeks, 18-22 g, male and female) were purchased from Shanghai Slake Laboratory Animal Co., Ltd and housed in Zhejiang University. The mice were maintained under pathogen-free conditions and acclimatized for 7 days before experiments. The animal experiments were conducted at the Zhejiang University. All animal experiments were conducted in compliance with the guide for the care and use of laboratory animals and approved by the Animal welfare and ethics committee, Zhejiang University (January 16, 2019, authorization number is No.18227). All the animals were anesthetized with ether, the blood samples were drawn from the eyes, and the mice were killed by the cervical dislocation method.

\section{Method}

\section{Experiment design}

The mice were randomly divided into five groups: blank control (BC), vaccine control (VC), PTFP-low dose (PTFP-L), PTFP-medium dose (PTFP-M), and PTFPhigh dose (PTFP-H) groups with 12 animals per group. The experimental procedure is represented as a schematic illustration in Figure 1. The mice (except the BC group) were subcutaneously immunized with $0.1 \mathrm{~mL}$ ND vaccine $\left(10^{6.0} \mathrm{EID}_{50} / 0.1 \mathrm{~mL}\right)$ on day 4 and boosted with the same dose on day 18. The mice in PTFP groups were continuously orally administrated with different doses of PTFP $(30,60$, and $120 \mathrm{mg} / \mathrm{kg}$ ) for 7 days (from day 1 to day 7 ), once a day. In addition, the oral administration 


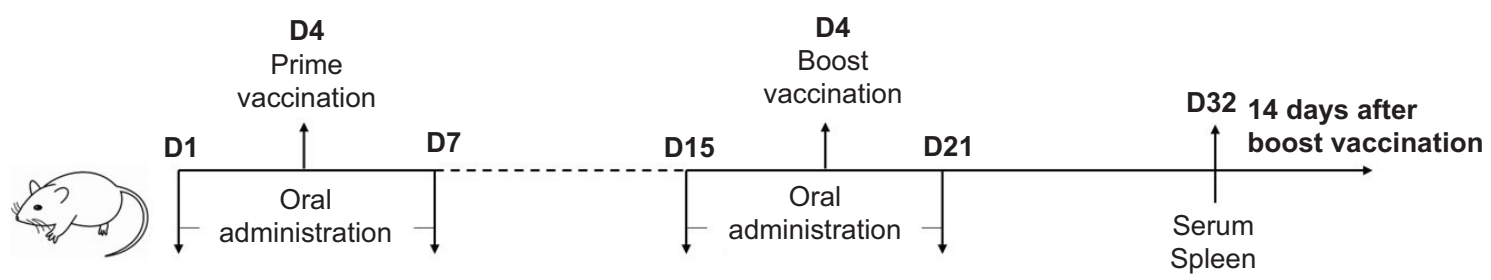

Figure 1. The schematic illustration of the vaccination and treatment schedule of the experiment.

procedure was performed again for 7 days from day 15 to day 21 . The mice in $\mathrm{BC}$ and VC groups were administrated with the same volume of physiological saline orally. About 14 days after the boost vaccination (at day 32), the mice were sacrificed, serum samples were collected, and spleens were harvested for subsequent immunological tests.

\section{Inhibition of hemagglutination assay (IHA)}

IHA titers against the NDV in the serum samples were carried out according to the IHA procedure as previously described (Wu et al., 2012; Wang et al., 2013; Liu et al., 2014; Zhang et al., 2014). Briefly, serum samples of different groups were collected at 14 days after boosting immunization and were heat-inactivated at $56^{\circ}$ for 30 minutes. The serum samples $(25 \mu \mathrm{L})$ were serially two-fold diluted using phosphate buffer saline (PBS) in a V-shaped microtiter plate. Later $25 \mu \mathrm{L}$ NDV antigen (4 hemagglutinating units (HAU) was added and incubated for 30 minutes at room temperature. Here, PBS was used as the negative control. Finally, $25 \mu \mathrm{L}$ of $1 \%$ chicken erythrocyte suspension was added, and the samples were reincubated for 30 minutes at room temperature. The IHA titer was expressed as the reciprocal value of the highest serum dilution, which completely inhibited the hemagglutination of chicken erythrocytes.

\section{Determination of antigen-specific antibodies in serum}

NDV-specific IgG and its isotypes (IgG1, IgG2a, and IgG2b) antibodies in the serum were measured on day 14 after boosting vaccination by ELISA (Wu et al., 2012; Sun et al., 2020). Briefly, 96 well ELISA plates were coated with $100 \mu \mathrm{L}$ NDV antigen $(0.5 \mathrm{HAU} / \mathrm{mL}$, carbonate solution, $\mathrm{pH}=9.6)$ per well at $4^{\circ}$ overnight. After washing thrice with PBS containing 0.5\% Tween 20, ELISA plates were blocked with PBS containing $1 \%$ bovine serum albumin and incubated for 2 hours at $37^{\circ}$. Then, the plates were washed thrice, and $100 \mu \mathrm{L}$ of serially diluted serum samples were added and reincubated for 2 hours at $37^{\circ}$. After rewashing thrice, the HRP-conjugated anti-mouse IgG (1:8000 diluted), IgG1 (1:6000 diluted), IgG2a (1:4000 diluted), or IgG2b (1:4000 diluted) antibodies were added into plates and incubated for 2 hours at $37^{\circ}$. Then ELISA plates were washed thrice, and $100 \mu \mathrm{L}$ of tetramethylbenzidine was added to the plates. After incubation for 15 minutes, $50 \mu \mathrm{L}$ of $2 \mathrm{M}$ sulphuric acid was added to stop the reaction. The optical density $(\mathrm{OD})$ was measured at $492 \mathrm{~nm}$ using a Bio-Rad 680 ELISA reader (Bio-Rad, Hercules, CA).

\section{Determination of spleen lymphocytes proliferation}

At 14 days after boosting immunization, splenocytes were harvested from the mice (Wang et al., 2016; Kumar et al., 2017; Huang et al.,2020; Lu et al.,2020; Yu et al., 2020; Gan et al., 2021). Briefly, the mice were sacrificed by cervical dislocation, and the spleens from different groups were aseptically separated. Later, they were crushed and passed through a 200-mesh sterile cell strainer, and the red blood cells were separated using cells lysis solution. The collected spleen lymphocytes $\left(5 \times 10^{6}\right.$ cells $\left./ \mathrm{mL}\right)$ were seeded into a 96-well plate with stimulated NDV antigen (0.0625 HAU/mL), Con A (final concentration $5 \mu \mathrm{g} / \mathrm{mL}$ ), or LPS (final concentration $10 \mu \mathrm{g} / \mathrm{mL}$ ), respectively. The cells incubated with RPMI-1640 medium were used as the negative control. After cultivation for 44 hours at $37^{\circ}$ in $5 \%$ carbon-di-oxide atmosphere, $50 \mu \mathrm{L}$ 3-(4,5-dimethylthiazol-2-yl)-2,5-diphenyl tetrazolium bromide (MTT; $2 \mathrm{mg} / \mathrm{mL}$ ) was added and further cultured for 4 hours at $37^{\circ}$. Subsequently, the supernatant was removed, and $150 \mu \mathrm{L}$ dimethyl sulfoxide (DMSO) was added. The absorbance at $570 \mathrm{~nm}\left(\mathrm{~A}_{570}\right)$ was measured by using a microplate reader. The stimulation index (SI) was calculated as the ratio of absorbance values of ND antigen, Con A, or LPS stimulated cells to untreated cells (negative control group) as shown in Equation (1).

$$
\mathrm{SI}=\frac{\mathrm{A}_{570}(\text { stimulated experimental group })}{\mathrm{A}_{570}(\text { negative control group })}
$$

\section{Determination of spleen lymphocytes cytokines by ELISA}

The spleen lymphocytes $\left(5 \times 10^{6}\right.$ cells $\left./ \mathrm{mL}\right)$ from different groups were incubated in 24-well plates and restimulated with NDV antigen $(0.0625 \mathrm{HAU} / \mathrm{mL})$. After incubation at $37^{\circ}$ for 72 hours, the productions of cytokines INF- $\gamma$ and IL-10 were detected in the supernatants collected after performing ELISA according to the manufacturer's instructions.

\section{Determination of NK cells activity}

The NK cells activity was determined as previously described (Xu et al., 2019; Zhang et al., 2020). In brief, the spleen lymphocytes $\left(1 \times 10^{7}\right.$ cells $\left.\left./ \mathrm{mL}\right)\right)$ of mice from 
different groups were collected as the effector cells, and the human leukemia K562 cells $\left(2 \times 10^{5}\right.$ cells $\left./ \mathrm{mL}\right)$ were used as the target cells. About $100 \mu \mathrm{L}$ of spleen lymphocytes were added into a 96-well plate with K562 cells $(100 \mu \mathrm{L})$ in the ratio of 50:1 (effector cells: target cells) and incubated for 20 hours at $37^{\circ}$. The MTT method was used to measure the cell viability of NK cells wherein 50 $\mu \mathrm{L}$ of MTT $(2 \mathrm{mg} / \mathrm{mL})$ was added to the plates and incubated for 4 hours. Then the supernatant was removed, DMSO was added, and the absorbance was determined at $570 \mathrm{~nm}$ by a microplate reader. The cell viability of NK cells was calculated according to Equation (2).

NK cells viability $=\frac{\mathrm{A} 570_{\mathrm{T}}-\left(\mathrm{A} 570_{\mathrm{S}}-\mathrm{A} 570_{\mathrm{E}}\right)}{\mathrm{A} 570_{\mathrm{T}}} \times 100 \%$

where $\mathrm{A} 570_{\mathrm{T}}$ : absorbance value of target cells control; A $570_{\mathrm{S}}$ : absorbance value of samples; and $\mathrm{A} 570_{\mathrm{E}}$ : absorbance value of effector cells control.

\section{Data analysis}

All data were expressed as the mean \pm standard deviation (SD). The statistical significance of differences was assessed by analysis of variance and Tukey's multiple comparisons. A probability value of $P<0.05$ was considered statistically significant.

\section{Results}

\section{Antibody responses}

\section{IHA antibody titers}

Figure 2 shows the significant increase in the IHA antibody titers in PTFP groups compared with the VC group

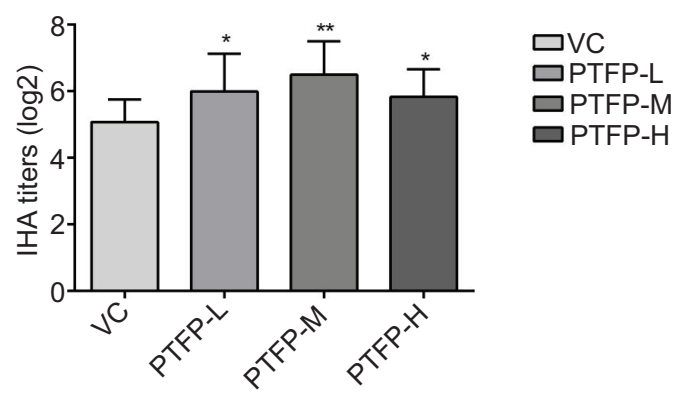

Figure 2. Effect of PTFP on IHA antibody response in the mice immunized with ND vaccine. The IHA antibody titers (log2) in serum from mice of different groups at 14 days after the boost immunization were determined by IHA assay. The values were presented as mean $\pm S D(n=12)$. Significant differences with the VC group were designated as ${ }^{*} P<0.05$ and ${ }^{* *} P<0.01$. IHA, inhibition of hemagglutination assay; VC, vaccine control; PTFP, Paulownia tomentosa flower polysaccharides; PTFP-L, PTFP-low dose; PTFP-M, PTFP-medium dose; PTFP-H, PTFP- high dose.
$(P<0.05)$. IHA antibody titers in PTFP-M group were higher than that of PTFP-L and PTFP-H groups and were not detected in the BC group (with no vaccination), whose data is not shown. The results demonstrated that PTFP, as an immunopotentiator, could promote IHA antibody responses in mice against ND-vaccine.

\section{Antigen-specific IgG and their isotypes (IgG1, IgG2a, and $\lg G 2 b)$ responses}

The antigen-specific IgG and isotypes (IgG1, IgG2a, and IgG2b) antibodies were determined by ELISA to further evaluate the humoral responses induced by PTFP in mice immunized with ND-vaccine. As shown in Figure 3A, PTFP in all groups significantly induced stronger antigen-specific IgG titers than the VC group $(P<0.05)$. IgG titers in the PTFP-M group were higher than the PTFP-L and PTFP-H groups. The results of IgG titers were consistent with IHA titers results (Figure 2).

The levels of antigen-specific IgG isotypes (IgG1, IgG2a, and IgG2b) in different groups were determined to investigate the effects of PTFP on T-helper (Th) 1 or Th2 immune responses. The IgG1 antibody was associated with a Th2-biased immune response. As shown in Figure 3B, IgG1 antibody titers in all the PTFP groups were significantly promoted compared with the BC group $(P<0.05)$. The levels of IgG1 antibody induced by the PTFP-M group were higher than the PTFP-L and PTFP-H groups. The results of IgG2a and IgG2b (Th1-associated) antibodies titers are shown in Figures $3 \mathrm{C}$ and 3D, respectively. IgG2a and IgG2b titers in all PTFP groups markedly increased (especially IgG2b) than the BC group. Moreover, IgG2a and IgG2b titers were induced by the PTFP-M group were higher than that in PTFP-L and PTFP-H groups. The results of IgG isotypes (IgG1, IgG2a, and IgG2b) in different groups had a similar trend to IgG titers (Figure 3A). In addition, ND-specific IgG and its isotypic (IgG1, IgG2a, and IgG2b) antibodies were not detected in the $\mathrm{BC}$ group (with no vaccination), and the data were not shown.

\section{Spleen lymphocytes proliferation}

To further investigate the effects of PTFP on cellular immune responses in mice against the ND-vaccine, spleen lymphocytes from mice were collected at 14 days after the boost vaccination, and the lymphocytes proliferation was stimulated with NDV-antigen, Con A, or LPS and determined. As shown in Figure 4A, after incubation with NDV antigen for 44 hours, lymphocytes proliferation in the PTFP groups (PTFP-L, PTFP-M, and PTFP-H groups) was significantly promoted compared with the VC groups $(P<0.05)$. The lymphocyte proliferation in the PTFP-M group was higher than the PTFP-L and PTFP-H groups. The result of lymphocytes proliferation with Con A-stimulation is shown in Figure 4B. The lymphocytes proliferation in the PTFP-L, PTFP-M, and PTFP-H 

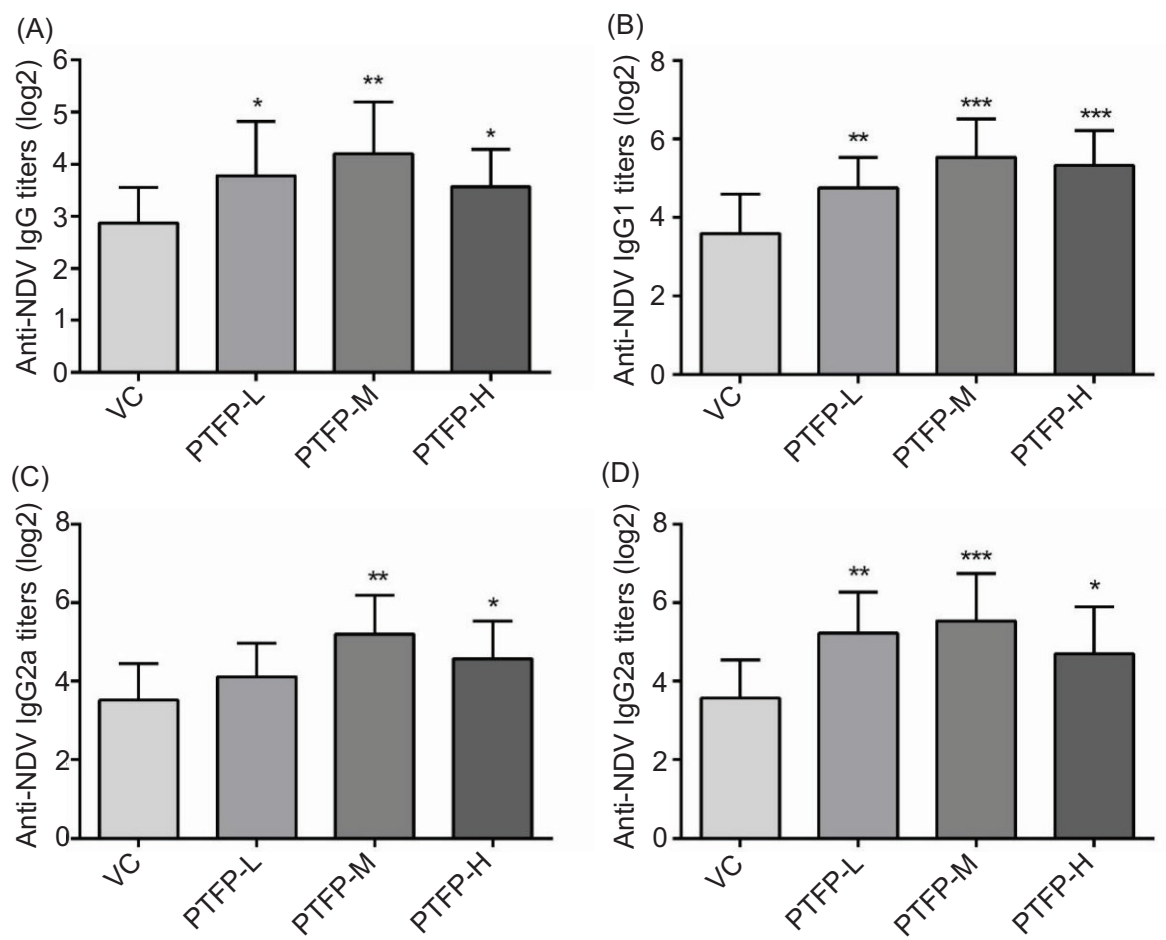

Figure 3. Effects of PTFP on antigen-specific antibodies responses in the mice immunized with ND vaccine. The antigen-specific $\lg G(A)$ and isotypes $\lg G 1(B), \lg$ Ga (C), and $\lg$ G2b (D) titers in the serum were measured by an indirect ELISA at 14 days after the secondary immunization. The values are presented as mean $\pm S D(n=12)$. Significant differences with VC group were designated as ${ }^{*} P<0.05$, ${ }^{* *} P<0.01$ and ${ }^{* *} P<0.001$. ND, Newcastle disease; NDV, ND-vaccine; VC, vaccine control; PTFP, Paulownia tomentosa flower polysaccharides; PTFP-L, PTFP-low dose; PTFP-M, PTFP-medium dose; PTFP-H, PTFP- high dose.

groups, especially the PTFP-M group, were improved compared with the VC group $(P<0.05)$. The trend of lymphocytes proliferation with LPS stimulation was similar to Con A stimulation (Figure 4C). All PTFP groups significantly promoted higher lymphocytes proliferation than the VC group $(P<0.05)$. The effect of lymphocytes proliferation with LPS stimulation in the PTFP-M group was better than those in the PTFP-L and PTFP-H groups.

\section{Spleen lymphocytes cytokines}

Spleen lymphocytes from immunized mice were collected and incubated with the NDV antigen for 72 hours 14 days after the second vaccination. The secretion of cytokines IFN- $\gamma$ and IL-10 were measured, and the results are shown in Figures $5 \mathrm{~A}$ and 5B. The levels of IFN- $\gamma$ in PTFP-L, PTFP-M, and PTFP-H groups were significantly increased compared with the VC group $(P<0.05)$. The IFN- $\gamma$ in PTFP-M groups was higher than that in the PTFP-L and PTFP-H groups. The IL-10 expressions in all PTFP groups were significantly higher than that in the VC group $(P<0.05)$, and IL-10 levels in PTFP-L and PTFP-M groups were higher vs. PTFP-H group.

\section{NK cells activity}

To further investigate the effect of PTEP on immunological enhancement, the cytotoxic activity of NK cells against human leukemia K562 cells was determined. The NK cells activity of splenocytes significantly increased in mice orally administrated with PTFP compared with the VC group (Figure 6) and was more strongly induced in PTFP-L and PTFP-M groups vs. the PTFP-H group.

\section{Discussion}

Recently, natural polysaccharides have been considered as novel immunopotentiators or immunomodulators because of their potent immune enhancement and low toxicity (Sun et al., 2018; Zeng et al., 2019; Zhao et al., 2020;). Numerous studies have demonstrated that polysaccharides extracted from Chinese medicinal herbs could enhance immune responses and promote the efficiency of vaccines by oral administration (Xie et al., 2012; Feng et al., 2013; Tang et al., 2019; Zhang et al., 2020). In our previous study, PTFP, extracted from P. tomentosa flower, had been proved to possess the immunomodulatory activity and enhance immune responses for orally administered ND-vaccine in chickens (Yang et al., 2019). Wang et al. (2019) have reported that PTFP as a new immunopotentiator or adjuvant could enhance humoral and cellular responses in chickens by injection administration. However, the mechanism of PTFP promoting 

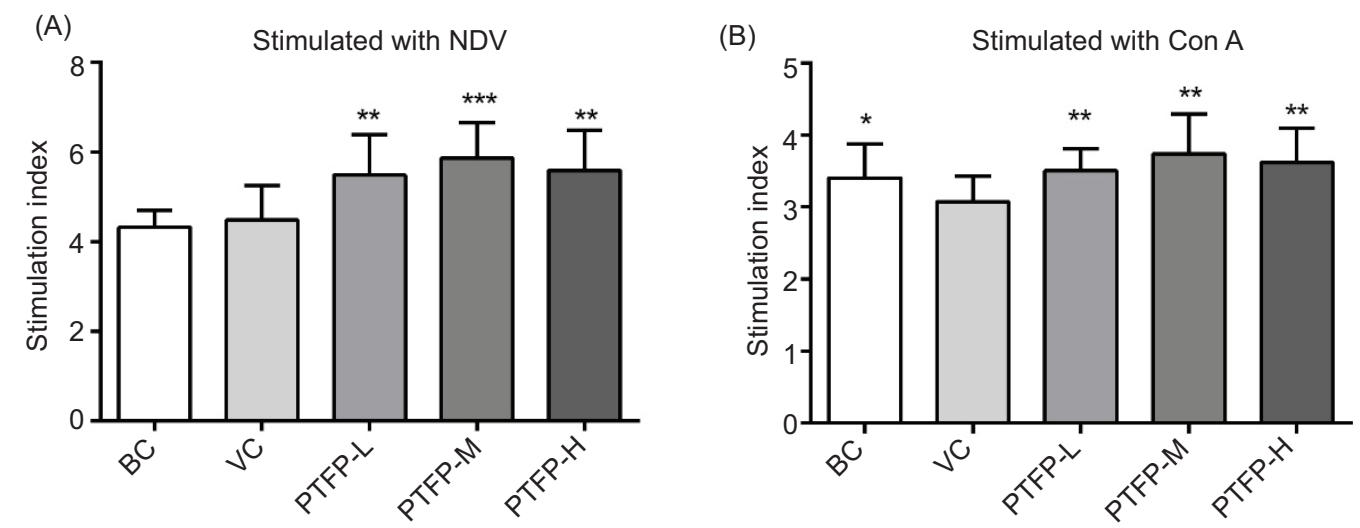

(C)

Stimulated with LPS

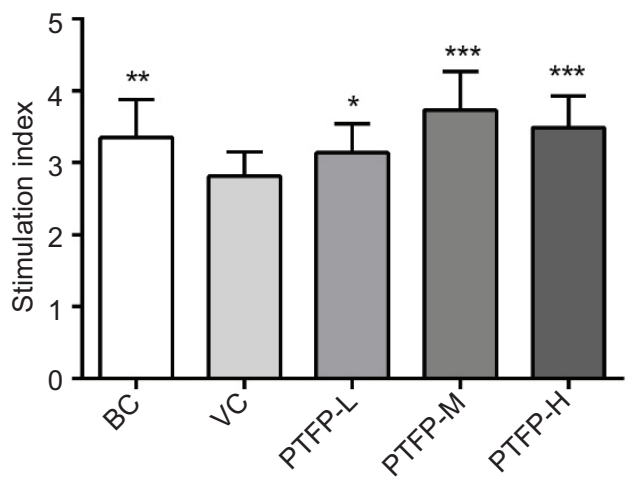

Figure 4. Effects of PTFP on spleen lymphocytes proliferation in the mice immunized with ND-vaccine. Splenocyte proliferation was detected by the MTT method after stimulation with ND antigen (A), Con A (B), or LPS (C) for 44 hours. The values are presented as mean $\pm S D(n=12)$. Significant differences with the VC group were designated as ${ }^{*} P<0.05$, ${ }^{* *} P<0.01$, and ${ }^{* * *} P<$ 0.001. ND, Newcastle disease; NDV, ND-vaccine; LPS, lipopolysaccharide; Con A, concanavalin A; BC, blank control; VC, vaccine control; PTFP, Paulownia tomentosa flower polysaccharides; PTFP-L, PTFP-low dose; PTFP-M, PTFP-medium dose; PTFP-H, PTFP-high dose.

(A)

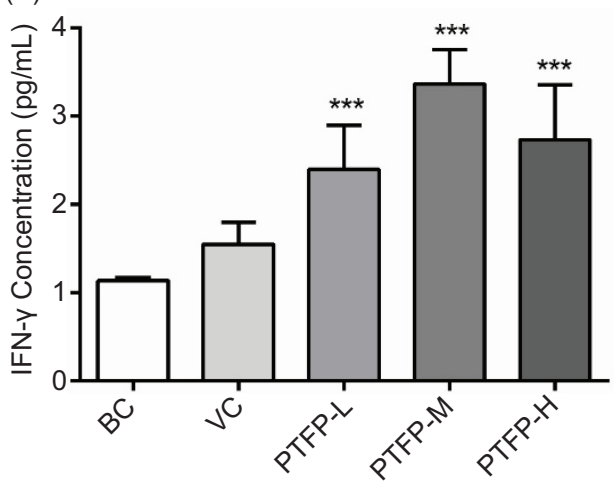

(B)

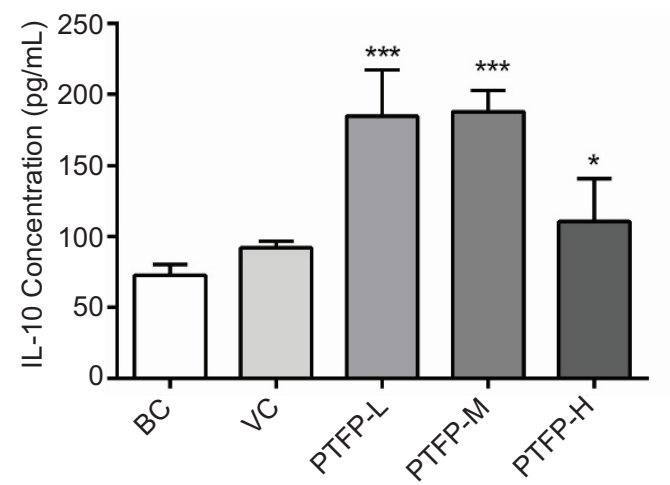

Figure 5. Effects of PTFP on spleen lymphocytes cytokines in the mice immunized with ND vaccine. The spleen lymphocytes from immunized mice were incubated with ND antigen for 72 hours. The IFN- $\gamma(A)$ and IL-10 (B) cytokines in supernatants of spleen lymphocytes were determined by ELISA. The values are presented as mean $\pm S D(n=12)$. Significant differences with VC group were designated as ${ }^{*} P<0.05$, ${ }^{* *} P<0.01$, and ${ }^{* * *} P<0.001$. IFN, interferon; IL, interleukin; BC, blank control; VC, vaccine control; PTFP, Paulownia tomentosa flower polysaccharides; PTFP-L, PTFP-low dose; PTFP-M, PTFP-medium dose; PTFP-H, PTFP- high dose. 


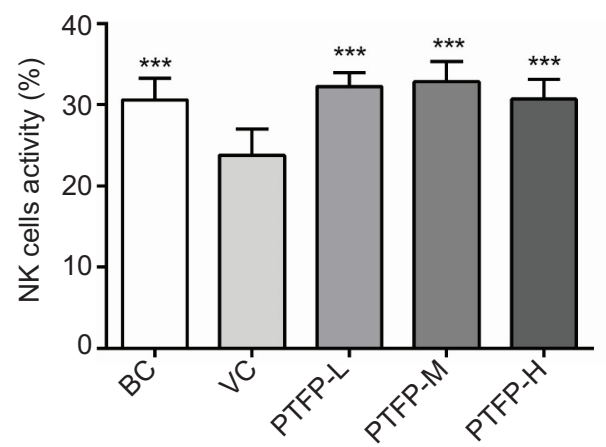

Figure 6. Effect of PTFP on NK cell activity in the splenocytes from the mice immunized with ND vaccine. Splenocytes from different groups were collected 14 days after the second immunization, and the NK cells activity was measured by MTT assay. The values are presented as mean $\pm S D(n=12)$. Significant differences with the VC group were designated as ${ }^{*} P<0.05$, ${ }^{*} P<0.01$, and ${ }^{* * *} P<0.001$. NK, natural killer; BC, blank control; VC, vaccine control; PTFP, Paulownia tomentosa flower polysaccharides; PTFP-L, PTFP-low dose; PTFP-M, PTFP-medium dose; PTFP-H, PTFP-high dose.

and regulating immune responses for ND vaccine by oral administration was still unexplored. Therefore, to further evaluate the immunomodulatory activity and investigate the mechanism of PTFP for ND-vaccine, mice were used as the model animal. Different doses of PTFP were orally administrated to it, and they were later immunized with ND vaccine.

NDV-specific antibody immune responses play an important and indispensable role in protecting the body from ND virus infection. The IHA was performed to determine the levels of specific antibodies against the hemagglutinin-neuraminidase protein (Yuan et al., 2020). Compared with the VC group, the mice orally administered with PTFP, especially with the PTFP-M dose, significantly increased the ND virus-specific IHA titers (Figure 2), indicating that PTFP with oral administration enhanced the antibody responses against the ND vaccine. In addition, the PTFP significantly promoted higher anti-NDV IgG antibody titers than the VC group (Figure 3A), which was consistent with the results of IHA in Figure 2. To further investigate the Th1 and Th2 immune responses induced by PTFP, antigen-specific IgG1, IgG2a, and IgG2b antibodies were determined. IgG1 antibody is associated with Th2-type immune, whereas IgG2a and IgG2b antibodies are associated with Th1-biased immune response (Feng et al., 2013). The PTFP promoted the productions of Th2-biased IgG1 antibody and IgG2a and IgG2b antibodies (Th1-biased) compared with the VC group (Figures 3B-D). These results suggested that PTFP induced a strong humoral immune response and elicited a mixed Th1 and Th2 response.
The stimulation of lymphocytes proliferation indicates the capacity of effective $\mathrm{T}$ and $\mathrm{B}$ lymphocytes immunity and hence are commonly used as an indicator to reflect the state of cellular immunity (Yang et al., 2008; Feng et al., 2013; Huang et al., 2013). Con A and LPS were cooperated to stimulate $\mathrm{T}$ and $\mathrm{B}$ lymphocytes proliferation, respectively (Yang et al., 2008; Wang et al., 2016). In Figure 4, the PTFP markedly increased the lymphocytes proliferation with the stimulation of NDV antigen, Con A, or LPS vs. the VC group. The result indicated that PTFP could effectively promote the NDV antigen-stimulated lymphocytes proliferation response, Con A-stimulated $\mathrm{T}$ lymphocytes proliferation, and LPSstimulated B lymphocytes proliferation.

Th1 lymphocytes mainly secreted IFN- - , IL-2, and IL-12 cytokines, whereas the Th2 cells predominantly produced IL-4, IL-6, and IL-10 cytokines (Liu et al., 2009). To further investigate the cellular immune response induced by PTFP, the spleen lymphocytes cytokines IFN- $\gamma$ and IL-10 were measured. IFN- $\gamma$, one of the main cytokines representing cellular immunity, could promote the antibody isotype switching to IgG2a and improve the differentiation and proliferation of Th1 cells (Zhang et al., 2020; Coutant et al., 2017). IL-10, a Th2-type cytokine, is considered an anti-inflammatory cytokine that regulates immune response (Courant et al., 2017). The PTFP both significantly improved the secretion of IFN- $\gamma$ and IL-10 cytokines when compared with the BC and VC groups (Figure 5A,B), indicating that PTFP both enhanced the Th1 and Th2 immune responses, which was consistent with the result of IgG1, IgG2a, and IgG2b antibodies (Figure 3B-D).

NK cells (the cytolytic effector lymphocytes of innate immunity) could recognize and eliminate virus-infected cells and tumor cells and are crucial in the innate immune system (Park et al., 2017; Xie et al., 2019). They act as a defense line against viral infections, and the activation of these cells plays a crucial role in regulating immune responses (Park et al., 2017; Xu et al., 2019). In Figure 6, compared with the VC group, PTFP significantly enhanced the NK cells activity by increasing the lysing of human leukemia K562 cells, indicating that PTFP with oral administration could markedly promote the activation of NK cells and improve immune responses.

Based on the results presented above, we investigated the effects of PTFP by oral administration on the mice immunized with ND vaccine and explained the mechanism of PTFP influences on the immune responses. First, the oral administration of PTFP enhanced the ND virus-specific IHA titers (Figure 2), increased the levels of anti-NDV IgG antibody titers (Figure 2A), and thereby improved the humoral immune response for the ND vaccine. Meanwhile, the PTFP promoted the productions of anti-NDV IgG1(Th2-type), IgG2a, and IgG2b (Th1-type) 
antibodies (Figures 3B-D), together with the secretions of IFN- $\gamma$ (Th1 cytokine) and IL-10 (Th2 cytokine; Figure 5), indicating that PTFP induced both the Th1 and Th2 immune responses. Moreover, it promoted the proliferation of $\mathrm{T}$ and $\mathrm{B}$ lymphocytes (Figure 4) and enhanced the cellular immune response. At the same time, the PTFP also activated the NK cells (Figure 6), thereby improving innate immunity. Hence, the oral administration of PTFP could enhance the humoral and cellular immune responses and initiate the innate immunity for ND vaccine in mice, which further demonstrated the possibility that PTFP could be the candidate of a new-type immunopotentiator in chickens. However, the values in the PTFP-H group were consistently lower than those in the PTFP-M group. No serious adverse effects of PTFP were observed after administration. It is necessary to design more groups to narrow the concentration gap between groups to find the best dose and study the side effects of PTFP in mice shortly. The cost of $P$. tomentosa flower is cheap, and the production process of PTFP is also simple. PTFP, as a new-type immunopotentiator, could play a significant role and have great developing space in poultry breeding.

\section{Conclusion}

In this study, to further investigate the immunomodulatory activity and explore the mechanism of PTFP for the $\mathrm{ND}$ vaccine, the mice were used as the model animals. Different doses of PTFP were orally administered to mice, and the ND vaccine was subcutaneously injected into them. Our finding demonstrated that orally administered PTFP enhanced the antigen-specific antibody immune responses and induced mixed Th1 and Th2 immune responses. Meanwhile, the PTFP also increased the proliferation of $\mathrm{T}$ and $\mathrm{B}$ lymphocytes, promoted the productions of IFN- $\gamma$ (Th1 cytokine) and IL-10 (Th2 cytokine), and improved the cellular immune response. In addition, it promoted the activation of NK cells thereby, improving innate immunity. Therefore, oral administration of PTFP could possess the immunomodulatory activity and improve immune responses by enhancing humoral immune response and cellular immune responses by inducing mixed Th 1 and Th 2 immune responses and promoting the innate immune response.

\section{Disclosure Statement}

The authors declare no competing interests.

\section{Funding}

This study was financially supported by the National Natural Science Foundation of China (31702286),
Qinglan Project of Jiangsu Province (2021), Key Research and Development Projects in Zhejiang Province and Anhui Province (2020C02032, S202104g01020071), Taizhou Science and Technology Supporting Agriculture Project (TN202006), Jiangsu Agri-animal Husbandry Vocational College Project (NSFPT201828), and Animal Medicine Science and Technology Innovation Team (NSF2021TC02).

\section{References}

Chen X., Han W., Wang G., Zhao X. 2020. Application prospect of polysaccharides in the development of anti-novel coronavirus drugs and vaccines. Int. J. Biol. Macromol.164:331-343. https:// doi.org/10.1016/j.ijbiomac.2020.07.106

Chen X., Yang H., Jia J., Chen Y., Wang J., Chen H., et al. 2021. Mulberry leaf polysaccharide supplementation contributes to enhancing the respiratory mucosal barrier immune response in Newcastle disease virus-vaccinated chicks. Poult Sci. 2021;100:592-602. https://doi.org/10.1016/j.psj.2020.11.039

Courant, T., Bayon, E., Reynaud-Dougier, H. L., Villiers, C., Menneteau M., Marche P.N., et al. 2017. Tailoring nanostructured lipid carriers for the delivery of protein antigens: physicochemical properties versus immunogenicity studies. Biomaterials. 136:29-42. https://doi.org/10.1016/j. biomaterials.2017.05.001

Dai B., Hu Z., Li H., Yan C., Zhang L. 2015. Simultaneous determination of six flavonoids from Paulownia tomentosa flower extract in rat plasma by LC-MS/MS and its application to a pharmacokinetic study. J. Chromatogr. B. 978-979:54-61. https://doi.org/10.1016/j.jchromb.2014.11.021

Feng H., Du X., Tang J., Cao X., Han X., Chen Z., et al. 2013. Enhancement of the immune responses to foot-and-mouth disease vaccination in mice by oral administration of a novel polysaccharide from the roots of Radix Cyathulae officinalis Kuan (RC). Cell. Immunol. 281(2):111-121. https://doi.org/10.1016/j. cellimm.2013.02.004

Gan T., Feng C., Lan H., Yang R., Zhang J., Li C., et al. 2021. Comparison of the structure and immunomodulatory activity of polysaccharides from fresh and dried longan. J. Funct. Foods. 2021;76:104323. https://doi.org/10.1016/j.jff.2020.104323

Huang R., Shen M., Yu Y., Liu X., Xie J. 2020. Physicochemical characterization and immunomodulatory activity of sulfated Chinese yam polysaccharide. Int. J. Biol. Macromol. 165:635644. https://doi.org/10.1016/j.ijbiomac.2020.09.213

Huang Y., Jiang C., Hu Y., Zhao X., Shi C., Yu Y., et al. 2013. Immunoenhancement effect of Rehmannia glutinosa polysaccharide on lymphocyte proliferation and dendritic cell. Carbohydr. Polym. 2013;96(2):516-521. https://doi. org/10.1016/j.carbpol.2013.04.018

Kumar S., Kesharwani S.S., Kuppast B., Bakkari M.A., Tummala H. 2017. Pathogen-mimicking vaccine delivery system designed with a bioactive polymer (inulin acetate) for robust humoral and cellular immune responses. J Control Release. 261:263-274. https://doi.org/10.1016/j.jconrel.2017.06.026 
Lee J.W., Seo K.H., Ryu H.W., Yuk H.J., Park H.A., Lim Y., et al. 2018. Anti-inflammatory effect of stem bark of Paulownia tomentosa Steud. in lipopolysaccharide (LPS)-stimulated RAW264.7 macrophages and LPS-induced murine model of acute lung injury. J. Ethnopharmacol. 210:23-30. https://doi.org/10.1016/j. jep.2017.08.028

Liu C., Ma J., Sun J., Cheng C., Feng Z., Jiang H., et al. 2017. Flavonoid-rich extract of paulownia fortunei flowers attenuates diet-induced hyperlipidemia, hepatic steatosis and insulin resistance in obesity mice by AMPK pathway. nutrients. 9(9):959. https://doi:org/10.3390/nu9090959

Liu Y., Jiao F., Qiu Y., Li W., Lao F., Zhou G., et al. 2009 .The effect of Gd@C82(OH)22 nanoparticles on the release of Th1/Th2 cytokines and induction of TNF-alpha mediated cellular immunity. Biomaterials. 30(23-24):3934-3945. http://ir.ihep.ac.cn/ handle/311005/239953

Liu Y., Wang L., Zhang Y., Zhang W., Chen X., Yang T., et al. 2014. Uniform-sized water-in-oil vaccine formulations enhance immune response against Newcastle disease and avian influenza in chickens. Int. Immunopharmacol. 2014;23(2):603-608. https://doi:org/10.1016/j.intimp.2014.10.011

Lu T., Hu F., Yue H., Yang T., Ma G. 2020. The incorporation of cationic property and immunopotentiator in poly (lactic acid) microparticles promoted the immune response against chronic hepatitis B. J. Control. Release. 321:576-588. https://doi. org/10.1016/j.jconrel.2020.02.039

Ma X., Bi S., Wang Y., Chi X., Hu S. 2019.Combined adjuvant effect of ginseng stem-leaf saponins and selenium on immune responses to a live bivalent vaccine of Newcastle disease virus and infectious bronchitis virus in chickens. Poult Sci. 98(9):3548-3556. https://doi.org/10.3382/ps/pez207

Park H., Wang D., Hong H., Shin K. 2017. Antitumor and antimetastatic activities of pectic polysaccharides isolated from persimmon leaves mediated by enhanced natural killer cell activity. J. Funct. Foods.37:460-466. https://doi.org/10.1016/j. jff.2017.08.027

Ren L., Zhang J., Zhang T. 2021. Immunomodulatory activities of polysaccharides from Ganoderma on immune effector cells. Food Chem. 340:127933. https://doi.org/10.1016/j. foodchem.2020.127933

Su C., Duan X., Liang L., Feng W., Zheng J., Fu X., et al. 2014. Lycium barbarum polysaccharides as an adjuvant for recombinant vaccine through enhancement of humoral immunity by activating Tfh cells. Vet. Immunol. Immunopathol. 158(1-2):98104. https://doi.org/10.1016/j.vetimm.2013.05.006

Sun B., Yu S., Zhao D., Guo S., Wang X., Zhao K. 2018. Polysaccharides as vaccine adjuvants. Vaccine. 36(35):52265234. https://doi.org/10.1016/j.vaccine.2018.07.040

Sun H., Fei L., Zhu B., Shi M. 2020. Quick and improved immune responses to inactivated $\mathrm{H} 9 \mathrm{~N} 2$ avian influenza vaccine by purified active fraction of Albizia julibrissin saponins. BMC Vet Res. 16(1):427. https://doi.org/10.1186/s12917-020-02648-1

Tang C., Sun J., Liu J., Jin C., Wu X., Zhang X., et al. 2019. Immuneenhancing effects of polysaccharides from purple sweet potato. Int. J. Biol. Macromol. 123:923-930. https://doi.org/10.1016/j. ijbiomac.2018.11.187
Wang J., Ge B., Li Z., Guan F., Li F. 2016. Structural analysis and immunoregulation activity comparison of five polysaccharides from Angelica sinensis. Carbohydr. Polym.140:6-12. https://doi. org/10.1016/j.carbpol.2015.12.050

Wang Q., Meng X., Zhu L., Xu Y., Cui W., He X., et al. 2019. A polysaccharide found in Paulownia fortunei flowers can enhance cellular and humoral immunity in chickens. Int. J. Biol. Macromol.130:213-219. https://doi.org/10.1016/j. ijbiomac.2019.01.168

Wang M., Meng X., Yang R., Qin T., Li Y., Zhang L., et al. 2013. Cordyceps militaris polysaccharides can improve the immune efficacy of Newcastle disease vaccine in chicken. Int. J. Biol. Macromol. 59:178-183. https://doi.org/10.1016/j. ijbiomac.2013.04.007

Wu Y., Wei W., Zhou M., Wang Y., Wu J., Ma G., et al. 2012. Thermalsensitive hydrogel as adjuvant-free vaccine delivery system for H5N1 intranasal immunization. Biomaterials. 33(7):2351-2360. https://doi.org/10.1016/j.biomaterials.2011.11.068

Xie F., Li Y., Su F., Hu S. 2012. Adjuvant effect of Atractylodis macrocephalae Koidz polysaccharides on the immune response to foot-and-mouth disease vaccine. Carbohydr. Polym. 87(2):17131719. https://doi.org/10.1016/j.carbpol.2011.09.080

Xie X., Ma L., Zhou Y., Shen W., Xu D., Dou J., et al. 2019. Polysaccharide enhanced NK cell cytotoxicity against pancreatic cancer via TLR4/MAPKs/NF-kappaB pathway in vitro/ vivo. Carbohydr. Polym. 225:115223. https://doi.org/10.1016/j. carbpol.2019.115223

Xu W., Fang S., Cui X., Guan R., Wang Y., Shi F., et al. 2019. Signaling pathway underlying splenocytes activation by polysaccharides from Atractylodis macrocephalae Koidz. Mol. Immunol. 111:1926. https://doi.org/10.1016/j.molimm.2019.03.004

Yang H., Zhang P., Xu X., Chen X., Liu Q., Jiang C. 2019. The enhanced immunological activity of Paulownia tomentosa flower polysaccharide on Newcastle disease vaccine in chicken. Biosci. Rep. 39(5): BSR20190224. https://doi.org/10.1042/ BSR20190224

Yang L., Hu Y., Xue J., Wang F., Wang D., Kong X., et al. 2018. Compound Chinese herbal medicinal ingredients can enhance immune response and efficacy of RHD vaccine in rabbit. Vaccine. 26(35):4451-4455. https://doi.org/10.1016/j.vaccine.2008.06.075

Yuan L., Wang Y., Li Z., Ma X., Cui X., Chi X., et al. 2020. Sunflower seed oil containing ginseng stem-leaf saponins (E515-D) is a safe adjuvant for Newcastle disease vaccine. Poult. Sci. 99(10):47954803. https://doi.org/10.1016/j.psj.2020.06.063

Yang Y., Xing R., Liu S., Qin Y., Li K., Yu H., et al. 2020. Chitosan, hydroxypropyltrimethyl ammonium chloride chitosan and sulfated chitosan nanoparticles as adjuvants for inactivated Newcastle disease vaccine. Carbohydr. Polym. 229:115423. https://doi.org/10.1016/j.carbpol.2019.115423

Yu Y., Song Q., Huang L., Shen M., Yu Q., Chen Y., et al. 2020. Immunomodulatory activities of sulfated Cyclocarya paliurus polysaccharides with different degrees of substitution on mouse spleen lymphocytes. J. Funct. Foods. 64:103706. https://doi. org/10.1016/j.jff.2019.103706

Zeng P., Li J., Chen Y., Zhang L. 2019. The structures and biological functions of polysaccharides from traditional Chinese 
herbs. Progress in molecular biology and translational science. 163:423-444. https://doi.org/10.1016/bs.pmbts.2019.03.003

Zhai L., Li Y., Wang W., Hu S. 2011a. Enhancement of humoral immune responses to inactivated Newcastle disease and avian influenza vaccines by oral administration of ginseng stem-andleaf saponins in chickens. Poult. Sci. 90(9):1955-1959. https:// doi.org/10.3382/ps.2011-01433

Zhai L., Li Y., Wang W., Wang Y., Hu S. 2011b. Effect of oral administration of ginseng stem-and-leaf saponins (GSLS) on the immune responses to Newcastle disease vaccine in chickens. Vaccine. 29(31):5007-5014. https://doi.org/10.1016/j. vaccine.2011.04.097

Zhang W., Gong L., Liu Y., Zhou Z., Wan C., Xu J., et al. 2020. Immunoenhancement effect of crude polysaccharides of
Helvella leucopus on cyclophosphamide-induced immunosuppressive mice. J. Funct. Foods. 69:103942. https://doi. org/10.1016/j.jff.2020.103942

Zhang W., Wang L., Liu Y., Chen X., Li J., Yang T., et al. 2014. Comparison of PLA microparticles and alum as adjuvants for H5N1 influenza split vaccine: adjuvanticity evaluation and preliminary action mode analysis. Pharm. Res. 31(4):1015-1031. https://doi.org/10.1007/s11095-013-1224-Z

Zhao Y., Yan B., Wang Z., Li M., Zhao W. 2020. Natural polysaccharides with immunomodulatory activities. Mini. Rev. Med. Chem. 20(2):96-106. https://doi.org/10.2174/138955751966619 0913151632 